\title{
Hemisphere differences in an auditory Stroop test
}

\author{
GILLIAN COHEN and MARYANNE MARTIN
}

\author{
Department of Experimental Psychology, University of Oxford, Oxford, England
}

\begin{abstract}
In an auditory Stroop test, right-handed subjects were required to judge the pitch of the following stimuli: two pure tones, one at a high frequency and one at a low frequency; two congruent words, "high," sung at the high frequency, and "low," sung at the low frequency; and two noncongruent words, "high" at low frequency and "low" at high frequency. A sequence of these stimuli was presented monaurally first to one ear, and then to the other. The Stroop effect (the difference between mean RT to congruent words, and mean RT to noncongruent words) was larger for right ear (left hemisphere) presentation. The same experiment was repeated dichotically with a competing message presented to the opposite ear. Again, the Stroop effect was larger for the right ear, and the ear differences were slightly more marked. The result is interpreted as reflecting hemispheric specialization for linguistic and nonlinguistic processing and a model of Stroop conflict in which response competition varies with the relative availability of the conflicting response.
\end{abstract}

Previous work has suggested that there exists some degree of hemispheric specialization for the analysis of linguistic and nonlinguistic auditory stimuli. In right-handed individuals, the left hemisphere $(\mathrm{LH})$ is superior for tasks requiring the analysis of verbal material; a right hemisphere $(\mathrm{RH})$ superiority has been demonstrated for the analysis of nonspeech stimuli, such as tones, melodies and patterns of clicks (Kimura, 1961, 1964). When hemispheric differences are manifested for a given task, it is usually impossible to distinguish between two explanations. Either both hemispheres can perform the task, but one is faster and more efficient than the other, or, alternatively, only one hemisphere can perform the task, so that when input reaches the inappropriate hemisphere it has to be transferred across the corpus callosum to the other hemisphere, and the loss of time and information during transit result in a performance decrement. Furthermore, it is quite possible that hemispheric asymmetry is due in some tasks to unequal efficiency, and in other tasks to callosal transfer. Clinical evidence, on the whole, suggests that language may be more completely lateralized than nonlinguistic functions.

With auditory stimulation, hemispheric asymmetry is related to ear of input. Although each ear has pathways projecting to both the contralateral and the ipsilateral hemisphere, an input via the contralateral pathway tends to occlude input arriving simultaneously via the ipsilateral path (Kimura, 1967). If, for example, a competitive input is presented to the left ear, the right-ear signal will be projected primarily to the LH. Verbal material therefore produces a

This work was supported by the Mental Health Research Fund and the Medical Research Council. The authors are most grateful to Jim Casey for technical assistance, and to Paul Dean, who sang the voice stimuli. right-ear advantage (REA) because it is projected to the more appropriate hemisphere. Nonverbal material, conversely, produces an LEA. With monaural stimulation, there exists no mechanism for blocking the ipsilateral path, so that signals are projected to both hemispheres. Although some neurophysiological evidence indicates that the contralateral path contains more fibres and contralateral stimulation produces larger evoked responses (Majkowski, Bochenek, Bochenek, Knapik-Fijalkowska, \& Kopec, 1971), ear differences have rarely been obtained with monaural stimulation. Choice reaction times have generally failed to reflect ear differences, or have shown effects attributable to attentional bias, rather than cerebral specialization (Simon, 1967). However, in memory tasks, Frankfurter and Honeck (1973) obtained an REA for sentence recall, and Bakker (1970) reported a monaural REA for recall of digits and an LEA for recall of sounds. He suggested that monaural ear differences can be elicited only if the task is sufficiently difficult.

An auditory version of the Stroop test appears to provide a suitable method for exploring hemispheric specialization for linguistic and nonlinguistic analysis, and for assessing the degree of interaction between the two. The classical version of the Stroop (1935) test demonstrates the interference which arises from naming the color in which a word is printed, when the word itself is the name of a different color. Analogous effects have been obtained in variations ot the Stroop test. For example, it is more difficult to count the number of stimulus items when the items themselves are digits that conflict with the counting response (Morton, 1969). Stroop-type interference is generated when the relevant physical attribute of a stimulus and its verbal label are incompatible. An explanation of 
this effect which has considerable experimental support has been offered by Morton and Chambers (1973) in terms of response competition. They suggest that interference is greater when the irrelevant attribute of the stimulus is analyzed faster than the relevant attribute, and the unwanted response is therefore available first.

An auditory version of the Stroop test can be devised in which the required response is a pitch judgment, the stimuli being either tones presented at two different frequency levels, high and low, or words, which may be congruent or noncongruent with the pitch at which they are presented. Ear differences can then be investigated within this paradigm. On the assumption that verbal analysis is carried out only, or more efficiently, within the $\mathrm{LH}$, then response competition when word and pitch are noncongruent would be more detrimental for stimuli presented to the right ear. When word and pitch are congruent, the L.H might also be expected to show more facilitation. Word meaning should produce less effect (either facilitation or interference) when the stimuli are presented to the left ear, because verbal analysis would be slower, or might not take place until after the stimuli were transferred across the corpus callosum to the LH. The verbal response might therefore not be available until after the pitch judgment was completed. If this model is correct, a substantially larger Stroop effect should be found for stimuli presented to the right ear. Although dichotic stimulation is usually necessary to produce such differences, a monaural condition was tested first for several reasons. Pilot testing showed that the task was quite difficult, and it was thought that a secondary task might depress performance excessively. And, since the task involved a memory load, it seemed possible that monaural asymmetries might emerge as in Bakker's (1970) experiment.

\section{EXPERIMENT I}

\section{Method}

Stimuli. Six stimuli were prepared. There were two pure tones-Tone $\mathrm{Hi}$ at $600 \mathrm{~Hz}$ and Tone $\mathrm{Lo}_{\mathrm{o}}$ at $400 \mathrm{~Hz}$, duration $800 \mathrm{msec}$. The four voice stimuli were two congruent words, $\mathrm{High} \mathrm{Hi}$

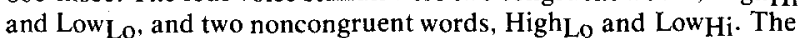
words were sung in a male voice. The singer attempted to reproduce the frequency of the tones and produced 10 examples of each of the required word-pitch combinations. From these, one example of each type which matched the tone most closely in frequency and duration was selected. The subjective judgment of two observers was checked against UV recordings. However, when the fundamental frequency of the voice stimuli had been isolated by filtering out the harmonics, it was found to be lower than its tone counterpart in each case. The fundamental frequency of the voice stimuli was: $\mathrm{High}_{\mathrm{Hi}}$ and $\mathrm{Low}_{\mathrm{Hi}}, 300 \mathrm{~Hz}$; and $\mathrm{High}_{\mathrm{Lo}}$ and Low Lo, $200 \mathrm{~Hz}$. This discrepancy was not considered to be damaging to the purposes of the experiment, partly because the subjective tone-voice match was good, and partly because the critical comparison in the experiment was not between tones and
Table 1

Mean Reaction Times in Milliseconds: Experiment 1

\begin{tabular}{lccc}
\hline & Tones & $\begin{array}{c}\text { Con- } \\
\text { gruent } \\
\text { Words }\end{array}$ & $\begin{array}{c}\text { Noncon- } \\
\text { gruent } \\
\text { Words }\end{array}$ \\
\hline Left Ear & 628 & 601 & 726 \\
Right Ear & 638 & 545 & 756 \\
\hline
\end{tabular}

words, but between the same stimuli when presented to different ears. The selected examples of each stimulus type were rerecorded to form a sequence of 36 stimuli, with each type recurring six times. Constraints superimposed on a random order equated both stimulus and response repetition across all types of stimulus.

Subjects. Eighteen subjects were tested. They were 10 male and 8 female students. A handedness questionnaire was administered and only subjects who were strongly right-handed were selected.

Procedure. Stimuli were presented monauraliy over Eagle International SE5 headphones, connected to a Sony Sterio $252 \mathrm{~W}$ tape recorder. Half the subjects had left-ear presentation first, and half had right-ear presentation first. The subjects were instructed to judge the pitch of each stimulus, ignoring the verbal content of the voice stimuli, and to respond by pressing one key for "high" and the other key for "low." They were urged to respond as fast and as accurately as possible. Half the subjects used the right hand for responses of "high," and the left hand for "low," and half had the opposite arrangement. Each subject first listened to the set of 6 stimuli, and then responded to a practice sequence. His responses were corrected by the experimenter if necessary, and practice continued until the subject was confident he could judge the stimuli correctly. The experimental sequence of 36 stimuli was then presented to one ear, and after a short pause, re-presented to the second ear. The subject was informed if he made an error, and error trials were replaced. An Advance timer was started by a bleep placed on the second channel of the tape at the onset of each stimulus, and was stopped by the keypress response. Reaction times were recorded in milliseconds.

\section{Results}

Mean RTs averaged over the 18 subjects are set out in Table 1 . The overall error rates were similar for both ears (left ear, $6.3 \%$; right ear, $6.7 \%$ ), and the number of errors occurring for each stimulus type was also the same for both ears.

A three-way analysis of variance (Subjects by Ears by Stimulus Types) was performed. The main effect of stimulus type was significant, $F(2,34)=8.2, \mathrm{p}<.01$, and the interaction of stimulus type with ear of presentation was also significant, $\mathrm{F}(2,34)=5.1$, $\mathrm{p}<.025$, reflecting the cross-over effect apparent in Table 1.

\section{Discussion}

The experimental results confirm the prediction that the Stroop effect is more marked in the right ear (LH). The difference between RTs for congruent words and RTs for noncongruent words measures the size of the Stroop effect, and this difference was $211 \mathrm{msec}$ in the right ear and $125 \mathrm{msec}$ in the left. A wilcoxon matched-pairs signed ranks test was employed to compare the size of the Stroop effect in left and right ears for each subject. Fifteen of the 18 subjects had a larger Stroop effect in the right ear, as shown in Table 2 , and the test yielded $\mathrm{T}=23$, $\mathrm{p}<.01$. It is worth noting that 2 mixed-handed 
Table 2

The Magnitude of the Stroop Effects for Individual Subjects in Milliseconds: Experiment 1

\begin{tabular}{crrr}
\hline Subjects & $\begin{array}{c}\text { Left } \\
\text { Ear }\end{array}$ & $\begin{array}{c}\text { Right } \\
\text { Ear }\end{array}$ & $\begin{array}{r}\text { Diffe- } \\
\text { rence }\end{array}$ \\
\hline 1 & 193 & 318 & 125 \\
2 & -30 & -27 & 3 \\
3 & -62 & 256 & 318 \\
4 & -75 & 189 & 264 \\
5 & 226 & 58 & -168 \\
6 & 250 & 278 & 28 \\
7 & 30 & 156 & 126 \\
8 & 319 & 384 & 65 \\
9 & 133 & 169 & 36 \\
10 & 213 & 190 & -23 \\
11 & 163 & 147 & -16 \\
12 & 129 & 208 & 79 \\
13 & 220 & 339 & 119 \\
14 & 118 & 128 & 110 \\
15 & 111 & 363 & 252 \\
16 & 25 & 54 & 29 \\
17 & 150 & 175 & 20 \\
18 & 40 & 425 & 385 \\
Mean & 125 & 211 & \\
\hline
\end{tabular}

subjects who were run on the experiment, but not included in the analysis, both showed a larger Stroop effect in the left ear.

The overall results are clearer when the ear differences for each of the three stimulus types are considered separately.

(1) Ear differences for tones. The 10-msec advantage for the left ear is in the expected direction, but is not significant. It may be that a significant advantage can be obtained only with dichotic, rather than monaural, stimulation. It has also been suggested (Day, Cutting, \& Copeland, Note 1) that variation in an irrelevant dimension may attenuate the advantage.

(2) Ear differences for congruent words. The 56-msec advantage for the right ear is significant by Newman-Keuls $(\mathrm{p}<.01)$.

RTs to congruent words are faster than to tones in the right ear by 93 msec $(p<.01)$, but in the left ear by only $27 \mathrm{msec}$ (n.s.). The finding that congruent words were judged faster than pure tones is difficult to interpret since the words and tones were not perfectly matched, but the superiority of the right ear suggests that congruence of word and pitch is facilitatory when the stimulus is presented to the $\mathrm{LH}$, and the pitch response is speeded by the coinciding verbal response.

(3) Ear differences for noncongruent words. Noncongruent words show a $30-\mathrm{msec}$ advantage for the left ear, which just fails to reach significance by the Newman-Keuls, test, but is significant by the Scheffé test. Right-ear stimulation has therefore produced markedly more facilitation for congruent words, and also slightly more interference for noncongruent words. As a result, noncongruent words were slower than congruent words by $211 \mathrm{msec}$ in the right ear and by $125 \mathrm{msec}$ in the left ear. Since both these differences are significant by Newman-Keuls, it is clear that some Stroop effect occurs in both hemispheres, although it is considerably larger in the left.

Interpretation of the results must therefore account for the occurrence of some Stroop difference in the RH. There are two possible explanations. Schmit and Davis (in press), who investigated hemispheric asymmetry in a visually presented color Stroop test, obtained the same pattern of results as the present experiment. RTs to noncongruent stimuli were elevated in both hemispheres, although to a greater extent in the LH. They reported that most of the Stroop effect occurring in the RH could be attributed to responses made with the right hand, and suggested that response conflict arose because the result of the nonlinguistic analysis carried out in the RH was contaminated by the conflicting linguistic analysis when the response was output by the LH. Left-hand responses could be output directly from the $\mathrm{RH}$, and so evaded the interference effect. This explanation was plausible for their experiment since the responses were individual finger movements, which are thought to be controlled by the contralateral hemisphere. In our experiment, the keypresses required gross whole-hand movements, which, according to Filbey and Gazzaniga (1969), can be mediated by either hemisphere. Inspection of our data revealed no difference in the size of the Stroop effect for left-hand and right-hand responses, so that an explanation locating the RH Stroop interference at the output stage is not tenable.

Another possible explanation derives from Morton's view of Stroop conflict as dependent on the relative speed of the two incompatible judgments. Stroop effects in the present experiment could arise whenever the verbal response was available prior to, or contemporaneously with, the pitch response. If the pitch analysis "raced" the verbal analysis, so that the pitch response could be output before the verbal response was available, neither facilitation nor interference effects would occur. Right-ear presentation would tend to favor the verbal response; the rapid verbal analysis in the $\mathrm{LH}$ might generate a verbal response ahead of the pitch response, which would be slower because the LH is inefficient at judging pitch, or because the stimulus had to be shuttled across to the RH for pitch analysis, or because the pitch analysis in the RH was handicapped by the weaker transmission of the ipsilateral path. It is arguable, therefore, that the verbal response would tend to "race" the pitch response and interact with it. Left-ear presentation would favor the pitch response, which would generally be concluded before the Stroop interactions could occur. To explain the RH Stroop effect, it is only necessary to postulate that on some 
small proportion of the trials the subject has difficulty reaching a decision as to the pitch of the stimulus, and the verbal response then "races" the delayed pitch response.

\section{EXPERIMENT II}

This tentative explanation of the results in terms of a "race" model of Stroop conflict is weakened by the fact that with monaural stimulation, signals are relayed to both hemispheres, and the contribution of each hemisphere to the final response is hard to assess. In order to minimize the contribution of the ipsilateral hemisphere, and to test the replicability of the findings, the experiment was repeated with a competitive input to the opposite ear, so that the signals were initially projected only to the contralateral hemisphere.

\section{Method}

Stimuli. Two new stimuli were added to the original ones. They were a high and a low version of a neutral word, intended to provide a baseline from which to asess the facilitating and interfering effects of the congruent and noncongruent words. The tones used in Experiment I had not provided an adequate baseline, partly because of the imperfect match and partly because the amount of information available in a pure tone and in a sung word is not equivalent. The new stimuli were formed from the word "bar," chosen as being semantically neutral and phonemically dissimilar to the other stimuli. They were sung in the same male voice as before. $\mathrm{Bar}_{\mathrm{Hi}}$ was matched to $\mathrm{High}_{\mathrm{Hi}}$ and Low Hi; BarLo was matched to Low $_{\text {Lo }}$ and HighLo. Six occurrences of each of these new stimuli were randomly interpolated into the original sequences, which was thereby extended to 48 trials.

For the competitive stimulation, a female speaker recorded two short stories, one by James Joyce and one by D. H. Lawrence, at normal reading speed.

Subjects. Eight strongly right-handed subjects were tested, five male and three female. About the same number of subjects had to be discarded from the experiment because they were unable to do the task and were still responding at chance level after $30 \mathrm{~min}$ of practice. The subjects who could do the task, on the other hand, all performed quite well and had no great difficulty. No obvious explanation has been found for this discrepancy.

Procedure. The stimulus sequence of $\mathbf{4 8}$ trials was presented four times to each subject ( 192 trials in all), with a change of ear between each presentation. Half the subjects were assigned to the order Right, Left, Right, Left, the other half to the order Left, Right, Left, Right. The same tape recorder, headphones, and timer were used with the same settings as in Experiment $I$. The output of a second Sony Stereo $252 \mathrm{~W}$ tape recorder playing the story tape was fed into the other earpiece of the headphones. Instructions and response requirements were exactly as in Experiment $I$. In addition, each subject was instructed to listen attentively to the short story, which was presented simultaneously to his other ear. He was told he would be asked detailed and specific questions about the story, and his replies would be scored. Each story continued through two presentations of the stimulus sequence, and recall was then tested. Ten questions covering material spread throughout the story were asked and scored. The subject then switched to the other story and continued with two further presentations of the stimulus sequence, after which recall of the second story was tested. Order of stories was balanced across subjects. Subjects who scored less than $60 \%$ on the story recall tests were dropped from the experiment. The session lasted $75 \mathrm{~min}$. Testing was preceded by a practice run using a different stimulus sequence and a different story.
Table 3

Mean Reaction Times in Milliseconds: Experiment 2

\begin{tabular}{lcccc}
\hline & Tones & $\begin{array}{c}\text { Neutral } \\
\text { Words }\end{array}$ & $\begin{array}{c}\text { Con- } \\
\text { gruent } \\
\text { Words }\end{array}$ & $\begin{array}{c}\text { Noncon- } \\
\text { gruent } \\
\text { Words }\end{array}$ \\
\hline Left Ear & 842 & 718 & 647 & 792 \\
Right Ear & 883 & 713 & 654 & 904 \\
\hline
\end{tabular}

\section{Results}

The mean RTs (eight subjects) are set out in Table 3. Error rates were $2.6 \%$ in the left ear and $4.4 \%$ in the right ear.

A three-way analysis of variance (Subjects by Ears by Stimulus Types) was performed. The main effect of stimulus type was significant, $F(3,21)=12.65$, $\mathrm{p}<.001$, and the interaction of stimulus type with ear of presentation was also again significant, $F(3,21)=$ $4.24, \mathrm{p}<.025$.

A two-way analysis of variance was performed to compare the size of the Stroop effect for left and right ears. The difference was significant with $F(1,15)=$ $9.25, \mathrm{p}<.01$. Individual Stroop effects are shown in Table 4.

Recall of the stories yielded a mean score of $82 \%$. correct. There was no difference between the two stories, and no difference in recall of material presented to the left or right ear. Subjectively, it seemed that close attention to the stories was required in order to be able to answer the questions accurately. While some switching of attention between ears cannot be ruled out, the story task provided a quite demanding competitive input.

A Newman-Keuls test was carried out to test the significance of differences between pairs of means, with the following results:

Ear differences. The left ear was faster for tones (by $41 \mathrm{msec}$ ) and for noncongruent words (by $112 \mathrm{msec}$ ). There were no ear differences for neutral words or congruent words.

Stimulus differences. Congruent words were responded to faster than neutral words on both ears. Noncongruent words were slower than congruent and slower than neutral on both ears. The difference

Table 4

The Magnitude of the Stroop Effects for Individual Subjects in Milliseconds: Experiment 2

\begin{tabular}{cccc}
\hline Subjects & $\begin{array}{c}\text { Left } \\
\text { Ear }\end{array}$ & $\begin{array}{c}\text { Right } \\
\text { Ear }\end{array}$ & $\begin{array}{c}\text { Diffe- } \\
\text { rence }\end{array}$ \\
\hline 1 & 204 & 214 & 10 \\
2 & 24 & 148 & 124 \\
3 & 65 & 191 & 126 \\
4 & 196 & 313 & 117 \\
5 & 36 & 9 & -27 \\
6 & 279 & 312 & 43 \\
7 & 168 & 353 & 185 \\
8 & 188 & 459 & 271 \\
Mean & 145 & 250 & \\
\hline
\end{tabular}


between noncongruent and neutral was $191 \mathrm{msec}$ for the right ear and $74 \mathrm{msec}$ on the left ear. All the differences are significant at $\mathrm{p}<.01$.

\section{Discussion}

The main finding of Experiment I was replicated. The Stroop effect was again larger for stimuli presented to the LH. But, whereas in Experiment I much of the asymmetry appeared to derive from the facilitation associated with congruent words, in Experiment II the asymmetry lies in the amount of interference generated by the noncongruent words. The left-ear advantage for tones, which failed to emerge with monaural stimulation, suggests that the competitive input succeeded in increasing the functional separation of the hemispheres. If the "race" model proposed as a possibie explanation of the results of Experiment I is correct, then the asymmetries evident in the monaural condition should be enhanced in Experiment II. The right-ear vulnerability to interference does appear to have increased, although the facilitation effect is equally evident for both ears. Several other factors have probably contributed to this result. The task of monitoring the short story ensures that verbal analysis is continually ongoing. This would make it more difficult to ignore the verbal content of the sung words, and, with verbal analysis predominantly carried out in the LH, interaction effects would be maximal in the LH. The puzzling element is that the $\mathrm{RH}$ performance is equally facilitated by word-pitch congruence, but is less impaired by conflict. Several subjects claimed that they adopted the congruent words stimuli, High $\mathrm{Hi}$ and Low Lo, as standards against which they matched all the others, and this strategy might account for the fast, symmetrical performance for congruent words.

In spite of the variations in the task introduced in Experiment II, the use of different subjects, and the use of diverse strategies by those subjects, the overall difference in the size of the Stroop effect for left- and right-ear presentation persists. This difference is best explained in terms of the relative specialization of the left and right cerebral hemispheres for linguistic and nonlinguistic processing, respectively, with this specialization producing a difference in the relative availability of verbal responses and pitch responses.

The paradigm employed in these experiments suggests several variations of the Stroop effect which could usefully be employed to explore hemispheric specialization for other auditory dimensions. For example, instead of requiring pitch judgments, judgments of intensity (loud, soft) or of speaker voice (male, female) could be examined when these dimensions were either congruent or in conflict with the verbal content of the stimuli.

The organization which renders the mechanism vulnerable to an auditory Stroop conflict of the type demonstrated is not necessarily a detrimental one. It must also serve to integrate linguistic and tonal information in listening to speech; it must underlie perception of languages like Thai, in which tone is a phonemic feature, and permit us to appreciate the interaction of words and melody in opera singing.

\section{REFERENCES}

BAKKER, D. J. Ear asymmetry with monaural stimulation: Relations to lateral dominance and lateral awareness. Neuropsychologia, 1970, 8, 103-117.

Filbey, R. A., \& Gazzaniga, M. S. Splitting the normal brain with reaction time. Psychonomic Science, 1969, 17, $335-336$

Frankfurther, A., \& Honeck, R. P. Ear differences in the recall of monaurally presented sentences. Quarterly Journal of Experimental Psychology, 1973, 25, 138-146.

Kimura, D. Cerebral dominance and the perception of verbal stimuli. Canadian Joumal of Psychology, 1961, 15, 166-171.

Kimura, D. Left-right difference in the perception of melodies. Quarterly Joumal of Experimental Psychology, 1964, 16, 355-358.

KimurA, D. Functional asymmetry of the brain in dichotic listening. Cortex, 1967, 3, 163-178.

MAJKoWSKI, J., BocheNeK, Z., BOCHENEK, W., KNAPIKFijalkowsKa, D., \& Kopec, J. Latency of average evoked potentials to contralateral and ipsilateral auditory stimulation in normal subjects. Brain Research, 1971, 25, 416-419.

Morton, J. Categories of interference: Verbal mediation and conflict in card sorting. British Journal of Psychology. 1969, 60, 329;345.

Morton, J., \& Chambers, S. M. Selective attention to words and colors. Quarterly Journal of Experimental Psychology, 1973, 25, 387.397.

Sснміт, V., \& Davis, R. The role of hemispheric specialization in the analysis of Stroop stimuli. Acta Psychologica, in press.

Simon, J. R. Ear preference in a simple reaction time task. Journal of Experimental Psychology, 1967, 75, 49-53.

STRoop, J. Studies of interference in serial verbal reactions. Journal of Experimental Psychology, 1935, 18, 643-662.

\section{NOTE}

1. Day, R. S., Cutting, J. E., \& Copeland, P. M. Perception of the linguistic and non-linguistic dimensions of dichotic stimuli. Haskins Laboratories Status Report, 27, 1971.

(Received for publication May 1, 1974; revision received July 25,1974 .) 